Original research article

\title{
Effect of gender, age, diet and smoking status on the circadian rhythm of ascorbic acid (vitamin C) of healthy Indians
}

\author{
Ranjana Singh ${ }^{\mathrm{a}, *}$, Abbas Ali Mahdi ${ }^{\mathrm{a}}$, Raj Kumar Singh ${ }^{\mathrm{a}, \mathrm{b}}$, Cathy Lee Gierke ${ }^{\mathrm{c}}$, \\ Germaine Cornelissen ${ }^{\mathrm{c}, * *}$ \\ a King George's Medical University, Biochemistry Department, Lucknow, India \\ ${ }^{\mathrm{b}}$ T S Misra Medical College and Hospital, Biochemistry Department, Lucknow, India \\ ${ }^{\mathrm{c}}$ University of Minnesota, Halberg Chronobiology Center, Minneapolis, USA
}

\section{A R T I C L E I N F O}

\section{Article history:}

Received 2 August 2017

Received in revised form 22 November 2017

Accepted 12 January 2018

Available online 1 February 2018

\section{Keywords:}

Ascorbic acid (vitamin C)

Circadian

Gender

Age

Diet (vegetarian)

Smoking

\begin{abstract}
A B S T R A C T
Background/Objectives: To determine effects of gender, age, diet, and smoking status on circadian rhythm characteristics of ascorbic acid (vitamin C).

Subjects/Methods: Ascorbic acid was measured spectrophotometrically in serum collected from 162 healthy volunteers (103 males and 59 females; 7-75 years) every 6 h for $24 \mathrm{~h}$ ( 4 samples). Data were analyzed by single and population mean cosinor. Effects of gender, age, diet (vegetarian vs. omnivore), and smoking status on the rhythm-adjusted mean (MESOR) and circadian amplitude were examined by multiple analysis of variance.

Results: A circadian rhythm is documented with statistical significance by population mean cosinor. In addition to effects of gender and age, the MESOR is affected by diet and smoking status. The circadian amplitude changes nonlinearly as a function of age. The circadian acrophase advances with increasing age.

Conclusion: The present observations confirm a definite circadian rhythm in ascorbic acid concentrations with significant effects of age, diet and smoking status in clinical health. Mapping the circadian rhythm of serum ascorbic acid in health can help explore its role in different pathophysiological conditions as predisease conditions may be characterized by alterations in the circadian amplitude and/or phase before there is a change in mean value.

(C) 2018 Faculty of Health and Social Sciences, University of South Bohemia in Ceske Budejovice. Published by Elsevier Sp. z o.o. All rights reserved.
\end{abstract}

\section{Introduction}

Vitamin C (ascorbic acid) is perhaps one of the most important single nutritional factors in terms of its influence on world history. Naval battles have been won or lost, based on the number of crewmembers sick with scurvy as well as on military prowess. Vitamin C, also known as L-ascorbic acid, is a water-soluble vitamin that is naturally present in some foods, added to others, and available as a dietary supplement. Humans, unlike most animals, are unable to synthesize vitamin C endogenously, so it is an essential dietary component (Li and Schellhorn, 2007).

\footnotetext{
* Author for correspondence: King George's Medical University, Biochemistry Department, 226003 Lucknow, India.

** Author for correspondence: University of Minnesota, Halberg Chronobiology Center, MMC 8609, 420 Delaware Street SE, Minneapolis MN 55455, USA.

E-mail addresses: ranjanasingh.2509@rediffmail.com (R. Singh), corne001@umn.edu (G. Cornelissen).
}

Oxidative stress generated by reactive oxygen species (ROS) is a critical component in the pathogenesis of a number of organic diseases and other neurodegenerative disorders, shown in human studies and supported by experimental models (Choudhry et al., 2012; Reddy and Beal, 2008). Reactivity of ROS can be terminated if the radical meets and reacts with an antioxidant molecule such as vitamin $\mathrm{C}$ or vitamin $\mathrm{E}$ ( $\alpha$-tocopherol), leading to neutralized or greatly reduced reactivity. Antioxidant molecules lose their antioxidant properties via this process and therefore must be constantly replenished or recycled. Oxidative stress is one of multiple processes that can lead to apoptosis in neurons (Mattson, 2000). Most endogenous ROS (including superoxide, hydrogen peroxide and the hydroxyl radical) are created by the electron transport chain during the production of ATP in the mitochondria (Eckert et al., 2003). Under normal circumstances, ROS are neutralized by antioxidant enzymes (superoxide dismutase, glutathione peroxidase, and catalase) and by antioxidants within the cell and the interstitial fluids (including vitamin C, vitamin E, and glutathione). A diet deficient in antioxidants can also lead to the impaired ability to repair neuronal 
DNA, which leaves the cells even more sensitive to oxidative damage (Kruman et al., 2002). Vitamin C is thought to be the most effective antioxidant in plasma, in part due to its water solubility and to the wide range of ROS that it can scavenge (Frei et al., 1990). If found to be protective against age-related and neurological diseases, vitamin C supplements could serve as an intervention of low cost and low toxicity. The elderly are at high risk of malnutrition, or at least subclinical malnutrition, for a number of reasons, including limited mobility, low income, institutionalization, reduced appetite, and poorer cognitive function. Preventing the depletion of antioxidant stores and maintaining healthy concentrations throughout the lifespan in order to improve the health of the population may be a more important strategy than trying to reverse ROS damage that has already occurred.

Vitamin $C$ is required for the biosynthesis of collagen, L-carnitine, and certain neurotransmitters. Vitamin C is also involved in protein metabolism (Li and Schellhorn, 2007). Collagen is an essential component of connective tissue, which plays a vital role in wound healing. Vitamin $\mathrm{C}$ is also an important physiological antioxidant (Frei et al., 1989), which has been shown to regenerate other antioxidants within the body, including alpha-tocopherol (vitamin E) (Jacob and Sotoudeh, 2002). Ongoing research examines whether vitamin C, by limiting the damaging effects of free radicals through its antioxidant activity, might help prevent or delay the development of certain cancers, cardiovascular disease, and other diseases in which oxidative stress plays a causal role. In addition to its biosynthetic and antioxidant functions, vitamin C plays an important role in immune function (Jacob and Sotoudeh, 2002) and improves the absorption of nonheme iron (Choudhry et al., 2012), the form of iron present in plant-based foods. Insufficient vitamin $C$ intake causes scurvy, which is characterized by fatigue or lassitude, widespread connective tissue weakness, and capillary fragility (Chambial et al., 2013; Li and Schellhorn, 2007). Although overt scurvy is now rare, there is evidence that subclinical vitamin $C$ deficiency is still quite common (Emadi-Konjin et al., 2005).

The intestinal absorption of vitamin $C$ is regulated by at least one specific dose-dependent, active transporter (Jacob and Sotoudeh, 2002). Cells accumulate vitamin C via a second specific transport protein. In vitro studies have found that oxidized vitamin C, or dehydroascorbic acid, enters cells via some facilitated glucose transporters, and is then reduced internally to ascorbic acid. The physiologic importance of dehydroascorbic acid uptake and its contribution to overall vitamin C economy is unknown.

Vitamin C exists in reduced (ascorbate) and oxidized forms (as dehydroascorbic acid), which are easily inter-convertible and biologically active. It thus acts as an important antioxidant. Due to its function as an antioxidant and its role in immune function, vitamin $C$ has been promoted as a means to help prevent and/or treat numerous health conditions. Diseases and disorders in which vitamin C might play a role include cancer (including prevention and treatment), cardiovascular disease, age-related macular degeneration (AMD) and cataracts. It has long been known that vitamin $C$ can be used as a protection against the common cold.

Under certain conditions, vitamin C can act as a pro-oxidant, potentially contributing to oxidative damage (Institute of Medicine, 2000). A few studies in vitro have suggested that by acting as a pro-oxidant, supplemental oral vitamin $C$ could cause chromosomal and/or DNA damage, and possibly contribute to the development of cancer (Institute of Medicine, 2000). However, other studies have not shown increased oxidative damage or increased cancer risk with high intakes of vitamin C (Institute of Medicine, 2000; Podmore et al., 1998). Blood is a commonly used biofluid for biomarker discovery. However, there are only few reports regarding the circadian nature of serum ascorbic acid in health and disease (Kushwaha et al., 2017; Singh et al., 2003, 2005, 2015). Moreover, the effect of gender, age, diet and smoking status on the MESOR (rhythm-adjusted mean) and circadian amplitude of ascorbic acid has not been studied in healthy Indians. The present study aims to fill this gap by quantifying serum ascorbic acid at different time points of the 24-h cycle and by assessing any effect of gender, age, diet and smoking status on the MESOR and circadian amplitude of ascorbic acid in healthy Indians.

\section{Materials and methods}

One hundred sixty-two clinically healthy Indians (103 males and 59 females, 7-75 years of age) volunteered for this study. The medical examination conducted when recruiting study participants ascertained the health status of all participants. Persons presenting with any known or clinically documented disease were excluded. Volunteers with a body mass index (BMI) less than 18.5 or more than $25.0 \mathrm{~kg} / \mathrm{m}^{2}$ were also excluded.

All subjects followed a 24-h synchronized social schedule with diurnal activity from about 06:00 to 22:00 and nocturnal rest. They were of equal socio-economic status (middle income), residing in the northern part of the country, around Lucknow. Most were medical students, staff members, and members of their families, who had been residing in the region for at least 2 years. At $25.50^{\circ}$, Lucknow is located just north of the Tropic of Cancer. There is seasonality in this part of the country and the average temperature ranges from $10^{\circ} \mathrm{C}$ to $45^{\circ} \mathrm{C}$. Informed consent was obtained from all individual participants included in this study, which was approved by the Institutional Review Board of King George's Medical University in Lucknow, India. The study was carried out in accordance with The Code of Ethics of the World Medical Association (Declaration of Helsinki).

Subjects were asked to refrain from taking any Vitamin C supplements, including any multivitamins with antioxidant activity before the study. All study participants were asked to avoid junk/fast food. All participants followed their usual daily routine, but abstained from strenuous activity, such as sports or other physical exercises on the dates of investigation. All took their usual (although not identical) meals 3 times daily: breakfast around 08:00, lunch around 13:30, and dinner around 21:00, without any change in their usual fluid intake. The burden of environmental temperature and pollution, if any, was common to all participants.

The volunteers were subdivided into 4 age groups: A (7-20 y), B (21-40 y), C (41-60y), and D (61-75 y), consisting of 42, 60, 35, and 25 subjects, respectively. The 7-20-year age group was included to span as wide an age range as possible without necessarily focusing on changes that may occur as a function of development and maturation. The dietary pattern of subjects in this age group was about the same as that of the other age groups.

Blood samples were collected in plain vials every $6 \mathrm{~h}$ for $24 \mathrm{~h}$ (4 samples) around 06:00, 12:00, 18:00 and 24:00. Serum was separated and ascorbic acid was measured spectrophotometrically (Natelson, 1971). Other biochemical variables of these volunteers have recently been published (Singh et al., 2016a,b).

Data from each subject were evaluated by conventional statistical analyses and by single and population mean cosinor procedures. Estimates of the MESOR (Midline Estimating Statistic Of Rhythm), a rhythm-adjusted mean, 24-h amplitude, a measure of half the predictable extent of daily change, and 24-h acrophase, a measure of the timing of overall high values recurring each day, were thus obtained (Cornelissen, 2014). Circadian rhythm characteristics were compared among the four age groups by parameter tests (Bingham et al., 1982). Multiple regression and multiple ANOVA, testing equality of group means, were used to examine any effect of gender, age, diet (vegetarian vs. omnivore), and smoking status on the MESOR and/or 24-h amplitude of ascorbic acid. The single and population mean cosinor analyses and parameter tests were performed using software developed at the 
Halberg Chronobiology Center, University of Minnesota. Conventional statistical analyses were carried out in R (R Core Team, 2017). A $P$-value of 0.05 or less was considered to indicate statistical significance.

\section{Results}

A circadian rhythm is invariably documented in each age group $(P<0.001)$. The results in Table 1 show the following: parameter tests show large changes in all circadian rhythm characteristics among the 4 age groups; both the MESOR and 24-h amplitude increase greatly between age groups $\mathrm{A}$ and $\mathrm{B}$ and between age groups $B$ and $C$, while they undergo a drastic decrease between age groups $C$ and $D(P<0.001)$. The 24 -h acrophase advances between age groups $A$ and $B$, and again between age groups $C$ and $D$ $(P=0.001)$ (Table 1$)$.

The nonlinear changes with age in the MESOR and 24-h amplitude of Vitamin C are illustrated in Fig. 1. Second-order polynomial models approximate well these changes as a function of age, indicating that the MESOR reaches a maximum around 46.9 years in women and around 46.4 years in men, and that the circadian amplitude reaches a maximum around 41.9 years of age in women and around 43.8 years of age in men. The progressive advance in the circadian acrophase with advancing age is illustrated in Fig. 2.

Multiple analyses of variance also find a large effect of age $(P<0.001)$, but no gender difference. A vegetarian diet, notably in age groups $\mathrm{B}-\mathrm{D}$, is found to be associated with a higher MESOR of Vitamin $C(P<0.001)$ (Fig. 3). Smoking status is found to be associated with a lower MESOR of Vitamin C $(P=0.033)$, an effect observed primarily among vegetarians. This is reflected by a statistically significant diet-smoking interaction $(P<0.001)$, (Fig. 4, left). An effect of smoking status of borderline statistical significance on the circadian amplitude of Vitamin $C$ is also found $(P=0.080)$, smokers having a smaller amplitude than non-smokers (Fig. 4, middle). As in the case of the MESOR, the difference is more pronounced among vegetarians, as shown by the statistically significant diet-smoking status interaction $(P=0.025)$, (Fig. 4, right).

\section{Discussion}

A circadian variation was found in serum ascorbic acid in healthy Indians of different ages. The MESOR increased significantly until about 46.5 years in both men and women. The circadian amplitude also started to decrease around 42 years of age, whereas the circadian acrophase advanced steadily throughout the lifespan, occurring in the oldest age group almost $9 \mathrm{~h}$ earlier than in the youngest age group. Such changes with age in the circadian amplitude and acrophase of ascorbic acid are also found in many other variables and are thought to reflect the aging process (Cornelissen and Otsuka, 2017).

With increasing age, circadian rhythms tend to become more labile, with a greater scatter of circadian acrophases, a diminished circadian amplitude, and a shift from the circadian to both ultradian (with periods shorter than a day) and infradian (with periods longer than a day) variation. This is reflected in Table 1 and Fig. 2, which illustrate the larger spread of phases in older subjects. Whether this "chronodisruption" stems from changes in the circadian rhythm of circulating melatonin cannot be answered herein since melatonin was not assessed. The fact that a reduction in circadian amplitude and a phase advance of core temperature were characteristic features after bilateral lesioning of the suprachiasmatic nuclei in the experimental laboratory, similar changes as those observed in older age groups, suggests the involvement of core clock genes in relation to aging (Cornelissen and Otsuka, 2017).

The present study reports for the first time statistically significant changes in all circadian parameters of serum ascorbic acid as a function of gender, age, diet, and smoking status assessed concomitantly. A literature search hardly found any studies by others reporting on the circadian variation of ascorbic acid. Loh and Wilson (1973) assessed plasma and taste threshold circadian rhythms of Vitamin C, based on 6 samples collected from 12 (5 M and 7 F) subjects 19-27 years of age, each sampled at 4-hourly intervals. Using the cosinor method, these authors reported a statistically significant circadian rhythm $(P=0.002)$ with a MESOR of $0.96 \pm 0.06 \mathrm{mg} / 100 \mathrm{ml}$, a 24 -h amplitude of $0.05 \mathrm{mg} / 100 \mathrm{ml}(95 \%$ confidence interval: $0.02-0.08)$, and a $24-\mathrm{h}$ acrophase of $-175^{\circ}$

Table 1

Age effects on circadian rhythm characteristics of ascorbic acid in healthy Indians.

\begin{tabular}{|c|c|c|c|c|c|c|c|c|c|}
\hline \multicolumn{10}{|c|}{ Population mean cosinor results } \\
\hline Age Group & $\mathrm{k}$ & MESOR & SE & 24 h Amplitude & SE & 24 h Acrophase & $(95 \% \mathrm{CI})$ & \multicolumn{2}{|c|}{ P-value $\left(\mathrm{H}_{0}: \mathrm{A}=0\right.$} \\
\hline A & 42 & 0.624 & 0.006 & 0.129 & 0.009 & $-289(19: 16)$ & $(-279,-299)$ & & \\
\hline B & 60 & 0.743 & 0.011 & 0.183 & 0.009 & $-242(16: 08)$ & $(-234,-249)$ & & \\
\hline $\mathrm{C}$ & 35 & 0.932 & 0.020 & 0.300 & 0.024 & $-232(15: 28)$ & $(-223,-243)$ & & \\
\hline $\mathrm{D}$ & 25 & 0.692 & 0.018 & 0.085 & 0.013 & $-159(10: 36)$ & $(-134,-182)$ & $<$ & \\
\hline \multicolumn{10}{|c|}{ Parameter tests } \\
\hline & \multicolumn{2}{|c|}{ MESOR } & & \multicolumn{2}{|c|}{$24 \mathrm{~h}$ Amplitude (A) } & \multicolumn{2}{|c|}{ 24-h Acrophase $(\phi)$} & \multicolumn{2}{|l|}{$(\mathrm{A}, \phi)$} \\
\hline Comparison & \multicolumn{2}{|c|}{$\mathrm{F}$} & $\mathrm{P}$ & $\mathrm{F}$ & ? & $\mathrm{F}$ & $\mathrm{P}$ & $\mathrm{F}$ & $\mathrm{P}$ \\
\hline A, B, C, D & \multicolumn{2}{|c|}{91.979} & $<0.001$ & 43.177 & & 20.570 & $<0.001$ & 41.987 & $<0.001$ \\
\hline $\mathrm{A}, \mathrm{B}$ & \multicolumn{2}{|c|}{74.265} & $<0.001$ & 23.914 & $<0.001$ & 43.428 & $<0.001$ & 31.080 & $<0.001$ \\
\hline $\mathrm{B}, \mathrm{C}$ & \multicolumn{2}{|c|}{85.659} & $<0.001$ & 32.375 & $<0.001$ & 2.352 & NS & 14.818 & $<0.001$ \\
\hline C, D & \multicolumn{2}{|c|}{78.095} & $<0.001$ & 43.589 & $<0.001$ & 11.349 & 0.001 & 41.265 & $<0.001$ \\
\hline A, D & \multicolumn{2}{|c|}{20.010} & $<0.001$ & 43.129 & $<0.001$ & - & - & 52.566 & $<0.001$ \\
\hline
\end{tabular}

Populations: A ( $<20$ years of age); B (21-40 years of age); C (41-60 years of age); D ( $>60$ years of age).

k: Number of subjects; MESOR: Midline Estimating Statistic Of Rhythm (a rhythm-adjusted mean) (mg/dl); SE: Standard Error; 24 h Amplitude (mg/dl); 24 h Acrophase (measure of timing of overall high values recurring each day) (negative degrees, with $360^{\circ} \equiv 24 \mathrm{~h} .0^{\circ}=00: 00$ ); $\mathrm{CI}$ : Confidence Interval.

Parameter tests (equality of MESOR, A, $\phi$ considered separately, and of (A, $\phi)$ considered jointly among populations being considered); test of equality of acrophases between two populations cannot be performed when they differ by more than $90^{\circ}$. 

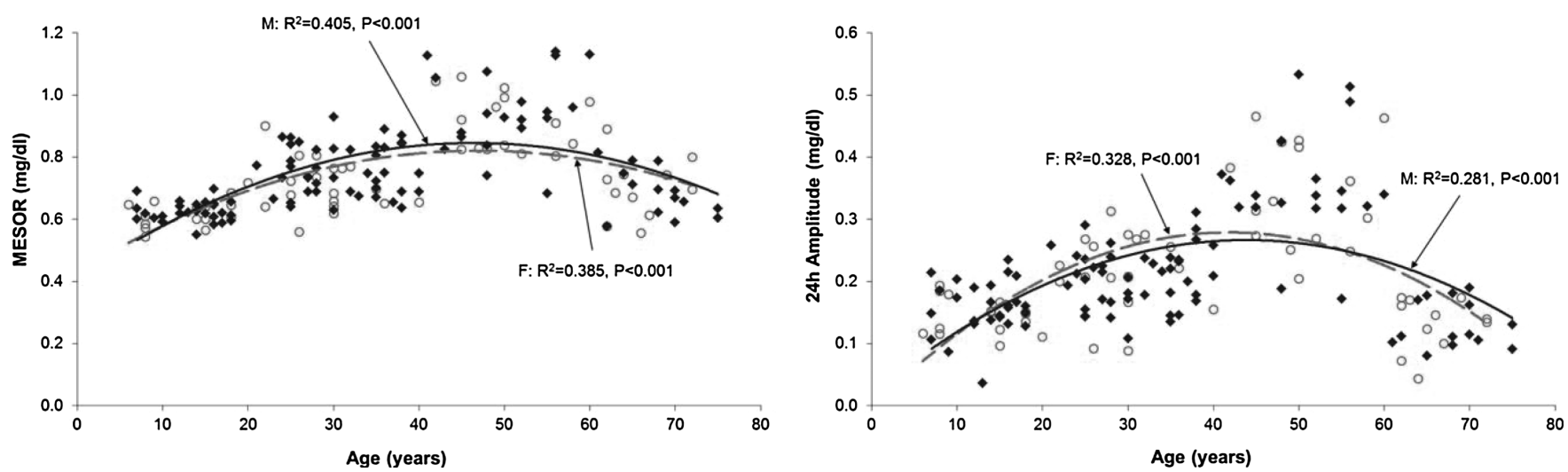

Fig. 1. Nonlinear relation with age of the MESOR (left) and circadian amplitude (right) of ascorbic acid, maximal values being reached between 40 and 50 years of age.

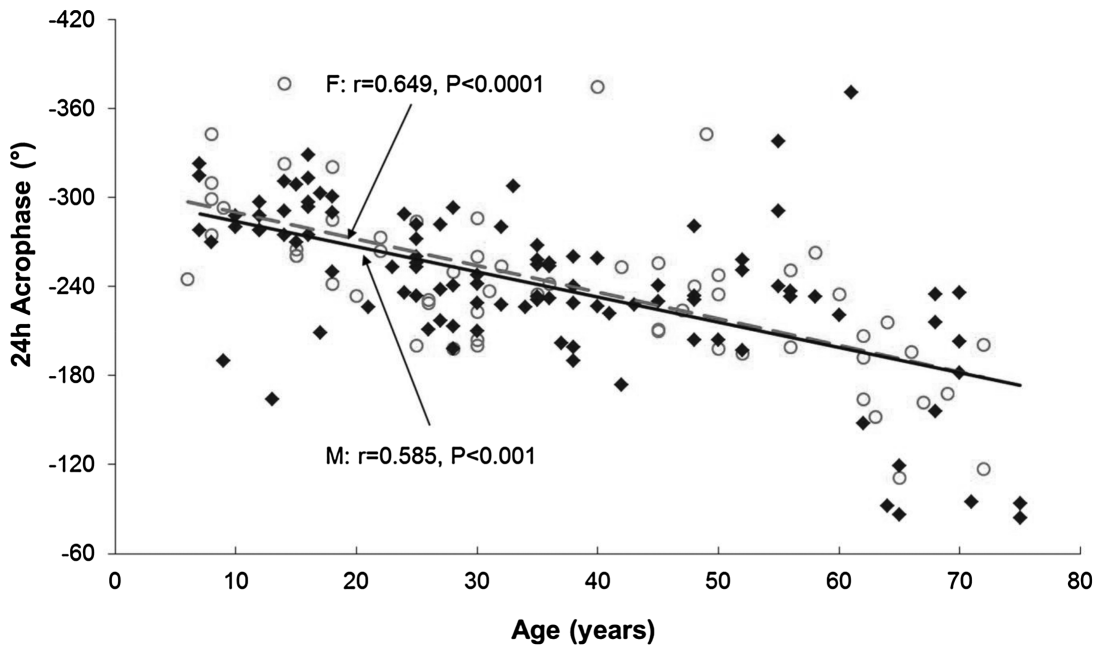

Fig. 2. Progressive advance of the circadian acrophase (timing of overall high values recurring each day) of ascorbic acid with increasing age.
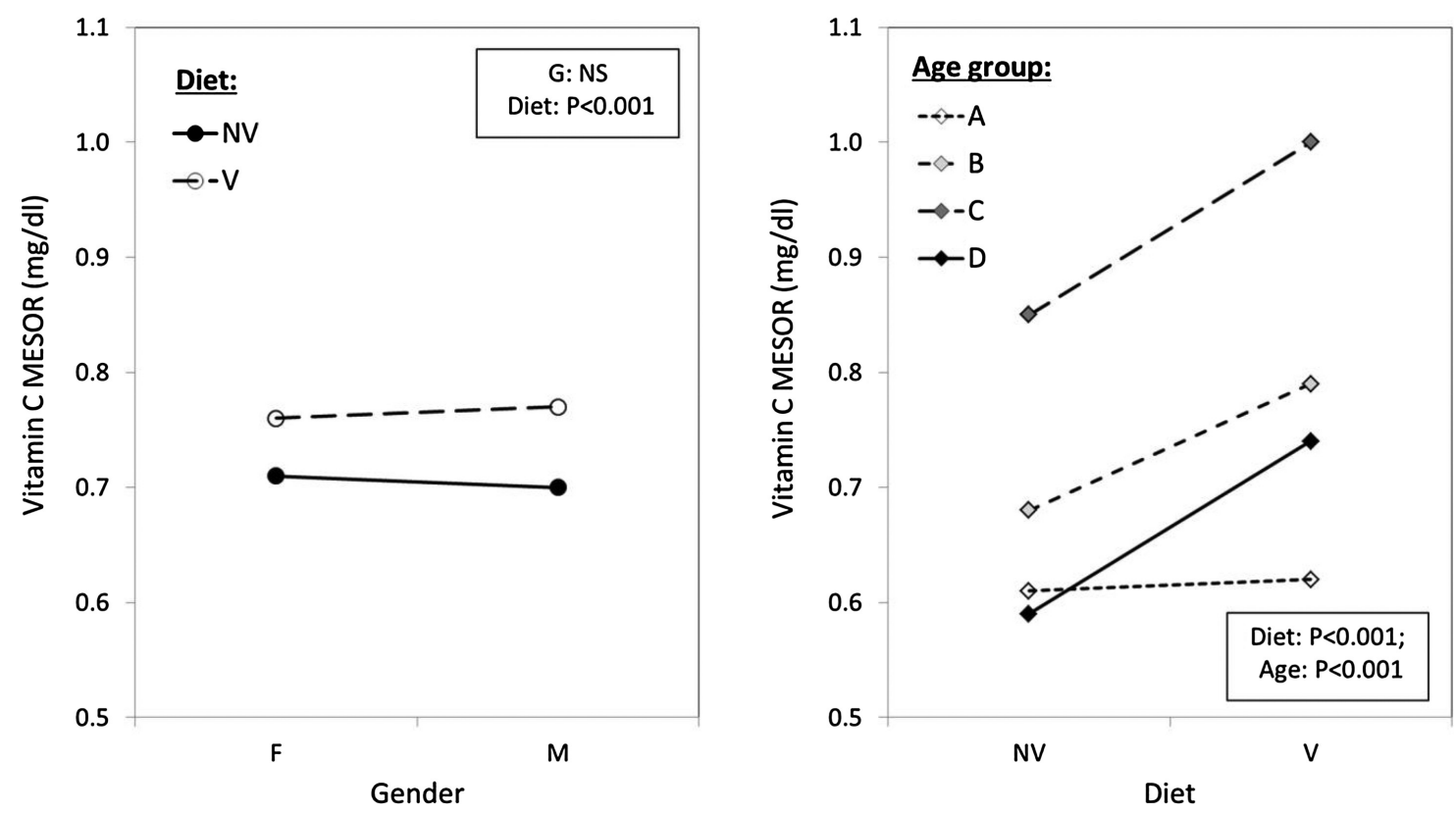

Fig. 3. A vegetarian diet, notably in age groups B-D, is found to be associated with a higher MESOR of Vitamin $C(P<0.001)$. 

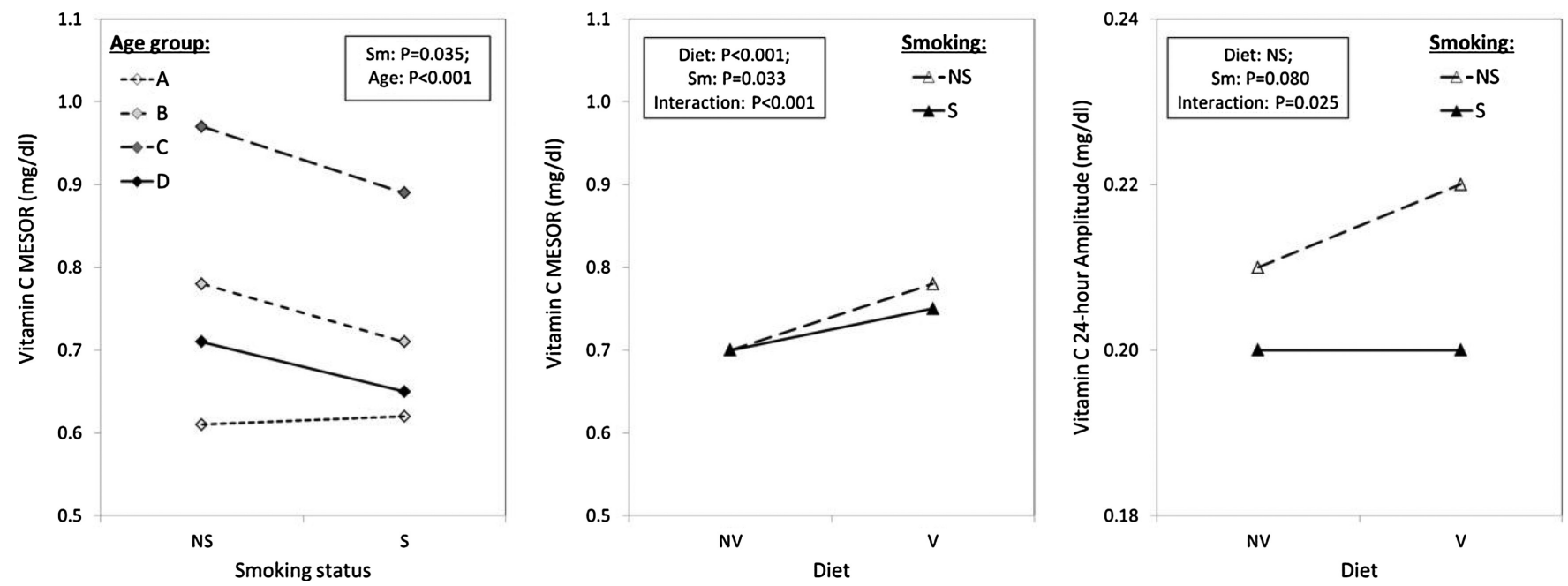

Fig. 4. Effect of smoking status on the MESOR and circadian amplitude of ascorbic acid, notably among vegetarians.

(95\% CI: $\left.-124^{\circ}--212^{\circ}\right)$ or $11: 40$ (95\% CI: 08:04-14:08). These results are similar to those observed in our investigation, except for an earlier circadian acrophase in the study of Loh and Wilson (1973), which is likely related to the different rest/activity schedule of the subjects (light from 04:00 to 20:00; dark from 20:00 to 04:00). By comparison, participants in our study were active from 06:00 to 22:00 and resting from 22:00 to 06:00. Accounting for this 2-h delay in the daily routine, the difference in circadian acrophase between the two studies is only about $2.5 \mathrm{~h}$ for subjects $21-40$ years of age. The circadian acrophase characterizing participants of the present investigation is in very good agreement with that of similar subjects involved in earlier studies conducted in India in both healthy people and in patients with liver cirrhosis, with peptic ulcers, or with kidney stones (Kushwaha et al., 2017; Singh et al., 2005, 2015, 2016a).

More evidence is needed for combined effects of gender, age, diet and smoking status on serum ascorbic acid concentrations in populations other than the one studied herein to allow results found in this study to be generalized. Some studies that investigated individual effects of gender, diet, and smoking status on vitamin C support our findings (Faure et al., 2006; Hercberg et al., 1998; Wei et al., 2016). The report that serum vitamin C was higher in female volunteers, and was not age related (Faure et al., 2006) may be accounted for by the fact that in this study, subjects ranged in age between 35 and 60 years of age. This age range corresponds to maxima in both MESOR and circadian amplitude of Vitamin $C$ in our study, when age-related changes are minimal. The discrepancy in gender differences may also be accounted for by the fact that in the study of Faure et al. (2006), men (50-60 years) were older than women (35-60 years). Herein, a vegetarian diet was associated with a higher MESOR of Vitamin C $(P<0.001)$, confirming the dietary significance of vitamin C. Smoking was found to be associated with a slightly lower MESOR of Vitamin C $(P=0.033)$, illustrating the negative role of smoking on vitamin $C$ concentration, and thus the necessity of this vitamin as supplement for smokers. Our results are consistent with those of other authors (Handelman et al., 1996; Marangon et al., 1998). The Ness team previously reported the relation between vitamin $\mathrm{C}$ and serum triglycerides; they also found an increase of HDL cholesterol in volunteers with high blood vitamin C concentrations (Ness et al., 1996). In our study as well, the MESOR of Vitamin $C$ was found to correlate positively with HDL cholesterol $(r=0.342, P<0.001)$.

Serum vitamins are not considered to be good markers of deficiency, and this is particularly true for vitamin C (Benzie, 1999).
The present study, however, aimed at assessing ascorbic acid results in clinically healthy Indians with normal body mass index. As expected, average concentrations of Vitamin C in our population averaged $0.75 \pm 1.4 \mathrm{mg} / \mathrm{dl}$, and ranged from 0.54 to $1.14 \mathrm{mg} / \mathrm{dl}$. These values are within the normal range of 0.41 to $2.00 \mathrm{mg} / \mathrm{dl}$ reported by Burtis et al. (2006).

Any further study of the circadian rhythmicity of ascorbic acid should address limitations of the present investigation. Denser sampling should be used. Taking samples every $6 \mathrm{~h}$ for $24 \mathrm{~h}$ is a bare minimum that only allowed the demonstration of a circadian rhythm on a population basis but not on an individual basis. Subjects studied herein were assumed to be 24-h synchronized and synchronized in phase to their daily routine of fixed rest/ activity and three daily meals. Additional information regarding their manility/serality (Horne and Ostberg, 1976) could easily be obtained by questionnaire. Around-the-clock measurements of core body temperature could serve as an added pertinent marker of the circadian system (Benloucif et al., 2005).

\section{Conclusion}

In summary, a definite circadian rhythm in ascorbic acid concentrations was documented in clinically healthy Indians of both genders and different ages. Circadian rhythm characteristics were shown to vary as a function of, diet, and smoking status. Mapping the circadian rhythm of serum ascorbic acid is needed to explore its role in different pathophysiological conditions, as predisease conditions may be characterized by alterations in the circadian amplitude and/or phase before there is a change in mean value.

\section{Conflict of interests}

The authors do not have any financial and personal relationships with other people or organizations that could inappropriately influence (bias) their work.

\section{Acknowledgements}

The authors are grateful to the late Professor Franz Halberg, known as the Father of Chronobiology, for his continuous guidance and support in continuation of chronobiological studies.

This work was supported by departmental grants (RS, RKS, AAM), and by the Halberg Chronobiology Fund (GC). 


\section{References}

Benloucif, S., Guico, M.J., Reid, K.J., Wolfe, L.F., L'hermite-Balériaux, M., Zee, P.C. 2005. Stability of melatonin and temperature as circadian phase markers and their relation to sleep times in humans. J. Biol. Rhythms 20,178-188.

Benzie, I.F.F., 1999. Vitamin C: prospective functional markers for defining optimal nutritional status. Proc. Nutr. Soc. 58, 469-476.

Bingham, C., Arbogast, B., Cornelissen, G., Lee, J.K., Halberg, F., 1982. Inferential statistical methods for estimating and comparing cosinor parameters. Chronobiologia 9, 397-439.

Burtis, C.A., Ashwood, E.R., Burns, D.E., 2006. Tietz Textbook of Clinical Chemistry and Molecular Diagnostics, 4th ed. Elsevier Saunders, St. Louis.

Chambial, S., Dwivedi, S., Shukla, K.K., John, P.J., Sharma, P., 2013. Vitamin C in disease prevention and cure: an overview. Ind. J. Clin. Biochem. 28, 314-328.

Choudhry, F., Howlett, D.R., Richardson, J.C., Francis, P.T., Williams, R.J., 2012. Prooxidant diet enhances beta/gamma secretase-mediated APP processing in APP/ PS1 transgenic mice. Neurobiol. Aging 33, 960-968.

Cornelissen, G., Otsuka, K., 2017. Chronobiology of aging: a mini-review. Gerontology 63, 118-128.

Cornelissen, G., 2014. Cosinor-based rhythmometry. Theor. Biol. Med. Model. 11, $16-40$

Eckert, A., Keil, U., Marques, C.A., Bonert, A., Frey, C., Schüssel, K., Müller, W.E., 2003. Mitochondrial dysfunction, apoptotic cell death, and Alzheimer's disease. Biochem. Pharmacol. 66 (8), 1627-1634.

Emadi-Konjin, P., Verjee, Z., Levin, A.V., Adeli, K., 2005. Measurement of intracellular vitamin $C$ levels in human lymphocytes by reverse phase high performance liquid chromatography (HPLC). Clin. Biochem. 38, 450-456.

Faure, H., Preziosi, P., Roussel, A.-M., Bertrais, S., Galan, P., Hercberg, S., Favier, A., 2006. Factors influencing blood concentration of retinol alpha-tocopherol, vitamin $\mathrm{C}$, and beta-carotene in the French participants of the SU.Vi.MAX trial. Eur. J. Clin. Nutr. 60, 706-717.

Frei, B., England, L., Ames, B.N., 1989. Ascorbate is an outstanding antioxidant in human blood plasma. Proc. Natl. Acad. Sci. USA 86, 6377-6381.

Frei, B., Stocker, R., England, L., Ames, B.N., 1990. Ascorbate: the most effective antioxidant in human blood plasma. Adv. Exp. Med. Biol. 264, 155-163.

Handelman, G.L., Packer, L., Cross, C., 1996. Destruction of tocopherols, carotenoids, and retinol in human plasma by cigarette smoke. Am. J. Clin. Nutr. 63, 559-565.

Hercberg, S., Preziosi, P., Briançon, S., Galan, P., Triol, I., Malvy, D., et al., 1998. A primary prevention trial using nutritional doses of antioxidant vitamins and minerals in cardiovascular diseases and cancers in a general population: the SU. VI.MAX study -design, methods, and participant characteristics. Control Clin. Trials 19 (4), 336-351.

Horne, J.A., Ostberg, O., 1976. A self-assessment questionnaire to determine morningness-eveningness in human circadian rhythms. Int. J. Chronobiol. 4 97-110.

Institute of Medicine. Food and Nutrition Board, 2000. Dietary Reference Intakes for Vitamin C, Vitamin E, Selenium, and Carotenoid Sexternal Link Disclaimer. National Academy Press, Washington DC.

Jacob, R.A., Sotoudeh, G., 2002. Vitamin C function and status in chronic disease. Nutr. Clin. Care 5, 66-74.
Kruman, I.I., Kumaravel, T.S., Lohani, A., Pedersen, W.A., Cutler, R.G., Kruman, Y., et al., 2002. Folic acid deficiency and homocysteine impair DNA repair in hippocampal neurons and sensitize them to amyloid toxicity in experimental models of Alzheimer's disease. J. Neurosci. 22, 1752-1762.

Kushwaha, R.S., Gupta, R.C., Sharma, J.P., Sharma, S., Singh, R.K., Cornelissen, G., 2017 Circadian periodicity of circulating plasma lipid peroxides, uric acid and ascorbic acid in renal stone formers. Ind. J. Clin. Biochem. 32, 220-224.

Li, Y., Schellhorn, H.E., 2007. New developments and novel therapeutic perspectives for vitamin C. J. Nutr. 137, 2171-2184.

Loh, H.S., Wilson, C.W., 1973. Vitamin C: plasma and taste threshold circadian rhythms: their relationship to plasma cortisol. Int. J. for Vit. \& Nutr. Res. 43, 355362.

Marangon, K., Herbeth, B., Lecomte, E., Paul-Dauphin, A., Grolier, P., Chancerelle, Y. et al., 1998. Diet, antioxidant status, and smoking habits in French men. Am. J. Clin. Nutr. 67, 231-239.

Mattson, M.P., 2000. Apoptosis in neurodegenerative disorders. Nat. Rev. Mol. Cell. Biol. 1, 120-129.

Natelson, S., 1971. Determination of ascorbic acid by 2,4- dinitrophenylhydrazine In: Thomas, C.C. (Ed.), Techniques of Clinical Chemistry. 3rd ed. Springfield, New York, pp. 165-166.

Ness, A.R., Khaw, K.T., Bingham, S., Day, N.E., 1996. Vitamin C status and serum lipids Eur. J. Clin. Nutr. 50, 724-729

Podmore, I.D., Griffiths, H.R., Herbert, K.E., Mistry, N., Mistry, P., Lunec, J., 1998. Vitamin C exhibits pro-oxidant properties. Nature 392, 559.

R Core Team, 2017. R: A Language and Environment for Statistical Computing. R Foundation for Statistical Computing, Vienna, Austria [online] [cit. 2017-1015]. Available from: https://www.R-project.org/.

Reddy, P.H., Beal, M.F., 2008. Amyloid beta, mitochondrial dysfunction and synaptic damage: implications for cognitive decline in aging and Alzheimer's disease. Trends Mol. Med. 14, 45-53.

Singh, R., Singh, R.K., Mahdi, A.A., Singh, R.K., Kumar, A., Tripathi, A.K., et al., 2003. Circadian periodicity of plasma lipid peroxides and other antioxidants as putative markers in gynaecological malignancies. In Vivo (Athens, Greece) 17 (6), 593-600.

Singh, R., Singh, R.K. Singh, R.K., Tripathi, A.K., Cornelissen, G., Schwartzkopff, O., et al., 2005. Chronomics of circulating plasma lipid peroxides, antioxidant enzymes and other related molecules in cirrhosis of liver. In memory of late Shri Chetan Singh. Biomed. \& Pharmacother. 59 (Suppl. (1)), S229-S235.

Singh, R., Singh, R.K., Masood, T., Tripathi, A.K., Mahdi, A.A., Singh, R.K., et al., 2015. Circadian time structure of circulating plasma lipid peroxides, antioxidant enzymes and other small molecules in peptic ulcers. Clin. Chim. Acta 451, 222 226.

Singh, R., Sharma, S., Singh, R.K., Cornelissen, G., 2016a. Circadian time structure of circulating lipid components in healthy Indians of different age groups. Ind. J Clin. Biochem. 31, 215-223.

Singh, R., Sharma, S., Singh, R.K., Mahdi, A.A., Singh, R.K., Lee Gierke, C., et al., 2016b. Effect of gender, age, diet and smoking status on chronomics of circulating lipid components in healthy Indians. Clin. Chim. Acta 459, 10-18.

Wei, J., Lei, G.H., Fu, L., Zeng, C., Yang, T., Peng, S.F., 2016. Association between dietary vitamin $\mathrm{C}$ intake and non-alcoholic fatty liver disease: a cross-sectional study among middle aged and older adults. PLoS One 11, e0147985. 